\title{
Chemische Quell- und Prozessindikatoren im Grundwasser
}

\author{
Tobias Licha $^{1} \cdot$ Wilhelm Püttmann ${ }^{2}$
}

Eingegangen: 9. September 2016 / Online publiziert: 28. September 2016 (c) Springer-Verlag Berlin Heidelberg 2016

Das vorliegende Themenheft verfolgt das Ziel, Raum für innovative Arbeiten zur Identifizierung und Anwendung hydro(geo)chemischer Indikatoren im Grundwasser zu bieten, welche das Potenzial haben, auf verschiedenen zeitlichen und örtlichen Skalen die Herkunft, die anthropogene Beeinflussung und die Wege des Grundwassers zu verfolgen. Immer dann, wenn sich ein Parameter entlang einer Fließstrecke ändert, birgt diese Zustandsänderung das Potenzial als Indikator genutzt werden. Dabei können Kontraste in physikalischen, physikochemischen oder chemischen Parametern, je nach Fragestellung und Untersuchungsgebiet, als Indikator fungieren. Alle Indikatoren sind jedoch dadurch charakterisiert, dass sie lediglich Änderungen detektieren und damit quantitative Aussagen aufgrund unbekannter Eingabefunktionen nicht direkt zulassen. Dies steht im Gegensatz zu Tracertests, in welchen eine bekannte Eingabefunktion mit einer Tracerdurchbruchskurve korreliert wird, um quantitative Ergebnisse (z. B. Porosität) abzuleiten. Sollen chemische Verbindungen als Indikatoren genutzt werden, müssen diese in erster Linie einen Konzentrationskontrast entlang einer Fließstrecke aufweisen. Weiterhin sollten die Verbindungen möglichst bei jeder Beprobung

Tobias Licha

tlicha@gwdg.de

Wilhelm Püttmann

puettmann@iau.uni-frankfurt.de

1 Abt. Angewandte Geologie, Geowissenschaftliches Zentrum der Universität Göttingen, Goldschmidtstraße 3, 37077 Göttingen, Deutschland

2 Abteilung Umweltanalytik, Institut für Atmosphäre und Umwelt der Universität Frankfurt, Altenhöferallee 1, 60438 Frankfurt/Main, Deutschland und in gut messbaren Konzentrationen gefunden werden. Gute Indikatoren sollten außerdem mit vertretbarem Aufwand chemisch-analytisch empfindlich nachweisbar sein. Aufgrund der großen Stoffvielfalt, des oft spezifischen Einsatzes und der breiten Eigenschaftspalette organischer Verbindungen ist es möglich, weitere Indikatorfunktionen zu nutzen. Sollen diese als Quellindikatoren fungieren, müssen Ihre Herkunft und Anwendungsgebiete bekannt und möglichst eindeutig zuzuordnen sein. Zeigen die Emissionen der Stoffe z. B. ein saisonales Verhalten auf, so kann dies als weitere Indikatorfunktion genutzt werden. Im Gegensatz zu Tracern, welche durch umweltrechtliche Vorgaben häufig auf eine geringe Bandbreite an Stoffen beschränkt sind, sind Indikatoren lediglich auf deren Anwesenheit und Konzentration im zu untersuchenden System beschränkt.

Um im Sinne der Nachhaltigkeit sicher zu stellen, dass die Quantität und Qualität dieser Ressource Grundwasser erhalten bleibt bzw. verbessert wird, müssen die physikalischen und chemischen Prozesse verstanden werden, die durch die Förderung im Aquifer ausgelöst werden. Die verstärkte Grundwasserentnahme induziert vielerorts eine zunehmende Infiltration von Oberflächenwasser. Da Oberflächengewässer heute insbesondere in Ballungsräumen mit zahlreichen polaren anthropogenen Spurenstoffen kontaminiert sind, besteht einerseits Bedarf nach Wissen bezüglich der biologischen Abbaubarkeit und dem Transport dieser Stoffe. Andererseits, können organische aber auch anorganische Wasserinhaltsstoffe, die nicht oder nur schlecht abbaubar bzw. mobil sind, genutzt werden, um die Infiltration von Oberflächenwasser in das Grundwasser abzuschätzen. Anthropogen eingetragene organische Spurenstoffe haben im Gegensatz zu anorganischen Wasserinhaltsstoffen keine natürlichen Hintergrundkonzentrationen, da sie ausschließlich durch menschliche Aktivitäten in die Umwelt gelangen. Dies bietet den Vorteil, dass Prozesse und Ein- 
tragsquellen unabhängig von geologischen Zusammenhängen mit hoher Sensitivität nachgewiesen werden können, als dies bislang mit herkömmlichen hydrochemischen Verfahren möglich ist. Die Suche nach geeigneten Indikatoren konnte in den letzten beiden Dekaden zahlreiche Erfolge verzeichnen (z. B. Gadolinium, 1H-Benzotriazol). Organische Substanzen müssen zwei wesentliche Eigenschaften in sich vereinen, um als potenzielle Indikatoren zu agieren: 1.) sie müssen polar genug sein, um im Wasserkreislauf mobil zu sein und 2.) ihre Abbaubarkeit muss unter den Randbedingungen des untersuchten Systems bekannt sein. Es mangelt nicht an Substanzen, die diese Eigenschaften erfüllen. Prädestiniert dafür sind z. B. iodierte Röntgenkontrastmittel oder der künstliche Süßstoff Acesulfam, welche im Grundwasser mobil, aber nur sehr schlecht abbaubar sind. Andererseits kann das leicht abbaubare Koffein genutzt werden, um den Anteil unbehandelten Abwassers in Grundwasserleitern zu quantifizieren, da es normalerweise bei der Abwasserbehandlung gut eliminiert wird. Dies setzt aber voraus, dass die Inputfunktion des Koffeins rekonstruierbar ist und damit der Indikator zum Tracer wird.

Erkenntnisse bezüglich der Nutzbarkeit von organischen und anorganischen Indikatoren für Fragestellungen, wie z.B. Einzugsgebietsabgrenzung, OberflächenGrundwasser-Interaktionen, Herkunft und Verweilzeit von Kontaminationen im Grundwasser, sowie die Bewertung der Effizienz wasserwirtschaftlicher Maßnahmen sind somit von herausragender Bedeutung, um insbesondere den Eintrag und die Verteilung von Abwasser in unserem Wasserkreislauf nachvollziehen zu können. Ein Beitrag zum Themenheft zeigt die aktuellen Möglichkeiten der Nutzung organischer Spurenstoffe für aktuelle Fragestellungen in der Hydrogeologie auf. Mit dem vorliegenden Themenheft werden weitere Studien (z. B. anhand von Nitrat) vorgestellt, die sich diesem Forschungsgegenstand widmen. 